\title{
Targeting glucose metabolism in chondrosarcoma cells enhances the sensitivity to doxorubicin through the inhibition of lactate dehydrogenase-A
}

\author{
GUOJUN HUA ${ }^{1}$, YUNPENG LIU ${ }^{1}$, XIANGYONG LI ${ }^{2}$, PEIRONG XU ${ }^{1}$ and YUCHUN LUO ${ }^{1}$ \\ Departments of ${ }^{1}$ Orthopaedics, and ${ }^{2}$ Hematology and Oncology, The 101st Hospital \\ of the People's Liberation Army, Wuxi, Jiangsu 214044, P.R. China
}

Received December 15, 2013; Accepted February 10, 2014

DOI: $10.3892 /$ or.2014.3156

\begin{abstract}
Chondrosarcoma is a malignant cartilage-forming cancer composed of cells derived from transformed cells that produce cartilage. Conventional chemotherapy and radiotherapy have very limited efficacy in patients with advanced chondrosarcoma. In the present study, we reported a novel therapeutic approach in the treatment of chondrosarcoma cells. We detected that lactate dehydrogenase-A (LDHA) is highly active in chondrosarcoma cells and chondrosarcoma patient samples compared with normal chondrocyte cell lines and primary human chondrocyte. Moreover, chondrosarcoma cells exhibited elevated levels of LDHA expression under doxorubicin treatment. To further explore the mechanisms, we generated doxorubicin-resistant cells from SW1353 chondrosarcoma cell line. Notably, the activity and expression of LDHA are upregulated in doxorubicin-resistant cells. Moreover, our data showed a strong correlation between glucose metabolism and doxorubicin resistance in chondrosarcoma cells; doxorubicinresistant cells displayed highly activated glucose metabolism and depended more on glucose supply. Finally, we reported a synergistic effect produced by incorporating doxorubicin with glycolysis inhibitors-oxamate in the combined treatment of chondrosarcoma cells in vitro and in vivo. In summary, the present study may aid in the development of new approaches using the combination of chemotherapeutic agents for the treatment of chondrosarcoma patients.
\end{abstract}

\section{Introduction}

Chondrosarcoma is a malignant cartilage-forming tumor which accounts for $20 \%$ of bone malignancies (1). The

Correspondence to: Dr Guojun Hua, Department of Orthopaedics, The 101st Hospital of the People's Liberation Army, Wuxi, Jiangsu 214044, P.R. China

E-mail: huaguojun101@163.com

Key words: glycolysis, inhibitor, chondrosarcoma, chemotherapy, doxorubicin, resistance conventional chondrosarcoma is associated with a significant rate of morbidity and the 10 -year survival rate is low $(29-83 \%)(1,2)$. In addition to surgical resection which is the primary treatment option for patients with chondrosarcoma, conventional chemotherapy and radiotherapy are still under investigation for treatment options (2-4). It is known that most chemotherapy drugs for the treatment of chondrosarcoma are associated with strong toxicities for normal cells, yet, tumor cells remain drug-resistant (3). Currently, chondrosarcomas are relatively chemotherapy- and radiotherapy-resistant. A recent study reported that the growth of chondrosarcoma cells can be inhibited by mTOR inhibitor in an in vivo syngeneic rat model (5), suggesting a putative chemotherapeutic approach for clinical applications.

Doxorubicin (Dox) is an antitumor drug that is used frequently in chemotherapy for a variety of solid tumors (6). Similar to other chemotherapeutic agents, the efficacy of doxorubicin treatment is limited by drug resistance (7). Despite considerable clinical responses initially, the majority of patients develop resistance to doxorubicin. Although the underlying mechanism of doxorubicin resistance is not fully understood, researchers have determined several factors that influence cellular doxorubicin toxicity. It has been reported that doxorubicin can induce ROS generation in various tumor cells $(8,9)$ and inhibition of P-glycoprotein by $20(\mathrm{~S})-\mathrm{Rh} 2$ attenuates adriamycin resistance (10).

Cancer cells, unlike their normal counterparts, use aerobic glycolysis with reduced mitochondrial oxidative phosphorylation for glucose metabolism (11). Therefore, the metabolic switch of cancer cells suggests that targeting metabolic pathway could be a selective approach not only to treat cancer patients, but also to override the chemoresistance. It has been reported that the combination of WZB117 which is a Glut1 inhibitor and cisplatin or paclitaxel displayed synergistic anticancer effects (12). Another GLUT1 inhibitor phloretin significantly enhanced daunorubicin's anticancer effects under hypoxia (13). Moreover, 3-BrPA which is a glycolysis inhibitor partially reversed the resistant phenotype and re-sensitized cells to oxaliplatin and 5-fluorouracil (14). Lactate dehydrogenase-A (LDHA) is one of the main isoforms of LDH expressed in breast tissue, controlling the conversion of pyruvate to lactate of the cellular glycolytic process. 
Studies have shown that the LDHA expression in cancer cells is associated with radiotherapy sensitivity (15). In addition, it has been reported LDHA contributed to paclitaxel resistance in breast cancer, inhibition of LDHA by oxamate overrides the chemoresistance (16).

In the present study, we reported a novel therapeutic approach in the treatment of chondrosarcoma cells. LDHA is highly active in chondrosarcoma cell lines compared with normal chondrocytes. Doxorubicin treatment induced the LDHA expression which contributes to the doxorubicin resistance. Meanwhile, the activity and expression of LDHA were upregulated in doxorubicin-resistant cells. Moreover, our data showed a strong correlation between glucose metabolism and doxorubicin resistance in chondrosarcoma cells. Doxorubicin-resistant cells displayed highly activated glucose metabolism and depended more on glucose supply. Finally, we reported a synergistic effect produced by incorporating doxorubicin with glycolysis inhibitors-oxamate in the combined treatment of chondrosarcoma cells in vitro and in vivo.

\section{Materials and methods}

Cell lines and cell culture. Hs 819.T and SW1353 human chondrosarcoma cells were purchased from ATCC. CHON-001 and $\mathrm{CHON}-002$ human normal chondrocyte cell lines were purchased from ATCC. Primary human chondrocyte was purchased from Promocell.com. All cells were cultured in RPMI-1640 supplemented with $10 \%$ FBS and $1 \mathrm{X}$ penicillinstreptomycin-glutamine (10378-016; Invitrogen) at $37^{\circ} \mathrm{C}$ in a humidified incubator with $95 \%$ air and $5 \% \mathrm{CO}_{2}$. All primary human chondrosarcoma patient specimens were obtained from patients undergoing surgery for tumor from 2009 to 2012 at the Cancer Research Center, The 101st Hospital of PLA, Wuxi, Jiangsu, China and stored in liquid nitrogen until analysis. All patients provided written informed consent. The study was approved by the Ethics Committee of the Cancer Research Center, The 101st Hospital of PLA, Wuxi.

Antibodies and reagents. Antibodies used in this study were purchased from: LDHA (Cell Signaling Technology \#2012); $\beta$-actin (Cell Signaling Technology \#4967); doxorubicin and oxamate were purchased from Sigma-Aldrich (St. Louis, MO, USA).

siRNA transfections. Transfection was performed using the Oligofectamine Transfection reagent (Invitrogen) according to the manufacturer's protocol. siRNA oligonucleotides for LDHA were purchased from Sigma, with a scrambled siRNA (Sigma) used as a control. Forty-eight hours after transfection, whole-cell lysates were prepared for further analysis.

Western blot analysis. Whole cells were lysed in 1X SDS sample buffer and resolved by electrophoresis using SDS-PAGE and transferred to nitrocellulose membranes. The membranes were probed with primary antibodies overnight, and then incubated with appropriate horseradish peroxidase conjugated secondary antibodies for $3 \mathrm{~h}$ followed by detection with a SuperSignal enhanced chemiluminescence kit (Pierce, Rockford, IL, USA). For sequential blotting, the membranes were stripped with Stripping Buffer (Pierce) and re-probed with proper antibodies.

Cell viability. Cell viabilities were determined using trypan blue dye exclusion assays. A total of $5 \times 10^{4}-1 \times 10^{5}$ cells/well were seeded in 12-well plates. Twenty-four hours later, the medium was replaced with different concentrations of doxorubicin or oxamate. After treatments with multiple concentrations of drugs, cells were trypsinized and resuspended in PBS. Viable cell numbers were determined by trypan blue staining.

Generation of doxorubicin-resistant cell line. SW1353 cells were treated with gradually increasing concentrations of doxorubicin in regular cell culture conditions for the selection of resistant cells. After successive treatments for up to 3 months, resistant cell clones were pooled and used for all subsequent experiments in the present study. The resistant cells were treated by doxorubicin each month for repeating selection.

Real-time PCR. RNA was extracted from cancer cells using the TRIzol reagent (Invitrogen, Carlsbad, CA, USA). The cDNA synthesis was performed using a SuperScript First-Standard Synthesis System for RT-PCR (Invitrogen) according to the manufacturer's protocol. Quantitative PCR analyses were performed using Assay-on-Demand primers and the TaqMan Universal PCR Master Mix reagent (Applied Biosystems, Foster City, CA, USA). The samples were analyzed using an ABI Prism 7700 Sequence Detection System (Applied Biosystems). The primers for q-PCR were: LDHA: forward, 5'-TGGAGTGGAATGAATGTTGC-3' and reverse, 5'-ATAGCCCAGGATGTGTAGCC-3'. The expression levels of $\beta$-actin were used to normalize the relative expression levels. Experiments were triplicated.

Glucose uptake assay. Cells were seeded in 12-well plates at $1 \times 10^{5}-3 \times 10^{5}$ cells/well. Culture media was collected at $48 \mathrm{~h}$ and stored at $-20^{\circ} \mathrm{C}$ until assayed. Glucose uptake was measured using an Amplex Red Glucose/Glucose Oxidase assay kit (Molecular Probes). Absorbance was measured at $563 \mathrm{~nm}$ using a SpectraMax M5 plate reader (Molecular Devices) and the results were normalized to the amount of total protein compared with the control cells.

Lactate production assay. Lactate production in the medium was detected by using a Lactate assay kit (BioVision). Results were normalized to the amount of total protein compared with the control cells.

LDHA activity assay. The total LDHA activity in cell lysates was examined according to the manufacturer's instructions of the LDH cytotoxicity assay kit (BioVision). Briefly, $2 \times 10^{5}$ cells were seeded in a 24-well plate one day before assaying and all samples were analyzed in triplicate. Then, cells were collected, washed and extracted for protein to measure LDHA activity. Results were normalized to the amount of total protein compared with the control cells.

Colony formation assay. For chondrosarcoma cell colony formation assay, 500 cells were seeded on a $10-\mathrm{cm}$ dish 
A

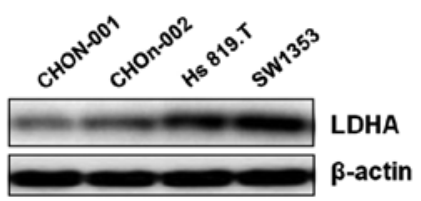

C
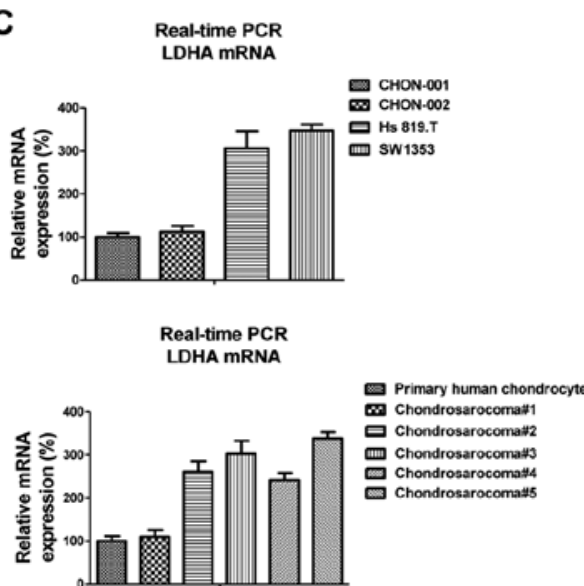

E

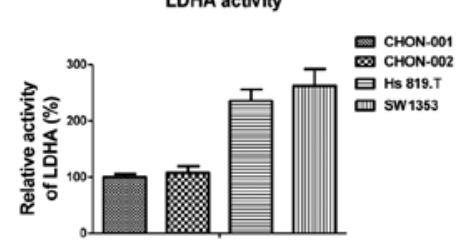

B

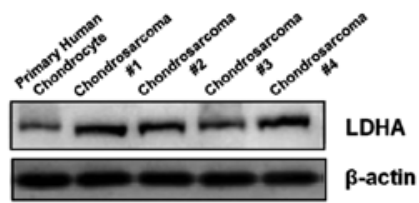

D

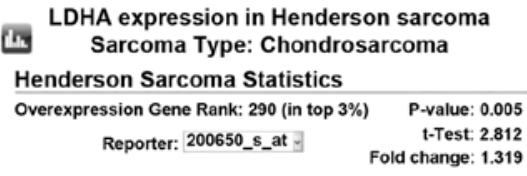

LDHA expression in Bittner sarcoma

Sarcoma Type: Chondrosarcoma

Bittner Sarcoma Statistics

Overexpression Gene Rank: 8898 (in top 46\%) P-value: 0.345 Reporter: 200650_s_at . t-Test: 0.437 Fold chane: 1.280
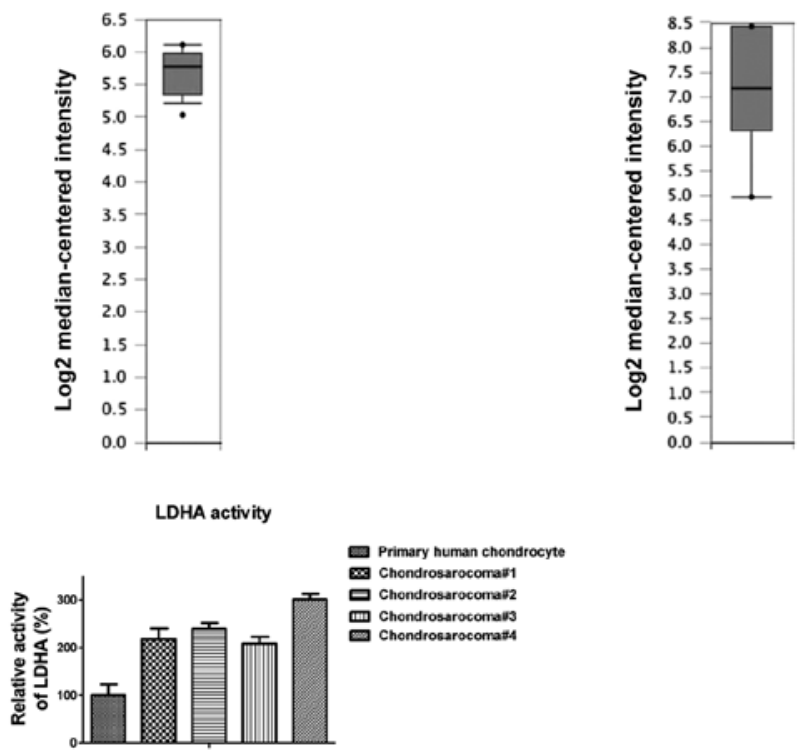

Figure 1. LDHA is upregulated in human chondrosarcoma cells. (A) Western blot analysis of human chondrocyte cell lines CHON-001 and CHON-002 and chondrosarcoma cell lines SW1353 and Hs 819.T. $\beta$-actin was a loading control. (B) Western blot analysis of human primary chondrocyte and tumor samples of chondrosarcoma patients. (C) mRNA expression of LDHA in the cell lines as indicated above. mRNA levels of LDHA were increased in chondrosarcoma cells compared with benign chondrocyte. (D) The relative expression of LDHA in chondrosarcoma analyzed from Oncomine.com. (E) Relative activity of LDHA in the above cells. The activities of chondrosarcoma cells were higher than chondrocyte. Columns, mean of three independent experiments; bars, SE.

with drugs in regular cell culture medium. The medium was refreshed every two days with drugs. Cells were grown for three weeks and the surviving colonies were stained with gentian violet after methanol fixation, and visible colonies $(>50$ cells) were counted. Colonies from randomly-selected image areas of three replicate wells were enumerated.

Animal experiments. Athymic BALB/c nude mice (5-8 weeks old) were housed in the Biological Resource Centre of the Department of Orthopaedics, The 101st Hospital of the People's Liberation Army. Mice were implanted subcutaneously in both sides of the flank with $3 \times 10^{6}$ chondrosarcoma cells without or with doxorubicin resistance, respectively. When the tumor reached a size of $>150 \mathrm{~mm}^{3}$, the mice were randomly divided into groups ( 8 mice per group) with the indicated treatments. Mice were weighed weekly and tumor diameters were measured with calipers twice per week for $>4$ weeks. Tumor progress was monitored by tumor size measurements every other day. All the experiments involving mouse models complied with both Chinese laws and the guidelines of the Ethics Committee of Jiangsu Institutes for Biological Sciences.
Statistical analysis. The unpaired Student's t-test was used for the data analysis. All data are shown as mean \pm standard error (SE). $\mathrm{P}<0.05$ was considered to indicate a statistically significant difference.

\section{Results}

LDHA is highly activated in chondrosarcoma cells. Cancer cells, unlike their normal counterparts, use aerobic glycolysis with reduced mitochondrial oxidative phosphorylation for glucose metabolism (11). Since LDHA is an important key enzyme of glycolysis, we first measured the LDHA expression in two malignant chondrosarcoma cell lines compared with benign human chondrocyte cell lines, CHON-001 and CHON-002. Notably, LDHA was significantly upregulated in chondrosarcoma cells (Fig. 1A). Consistently, the expression of LDHA in chondrosarcoma patient tumor samples was also upregulated compared with primary human chondrocyte (Fig. 1B), indicating the elevated LDHA expression might be the target for chondrosarcoma chemotherapy. Real-time PCR results showed the mRNA of LDH-A was also increased in chondrosarcoma cells (Fig. 1C). Bioinformatics research 
A

Hs 819.T

Dox

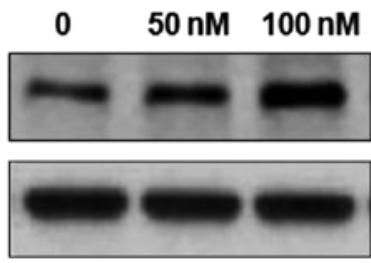

LDHA

$\beta$-actin

B

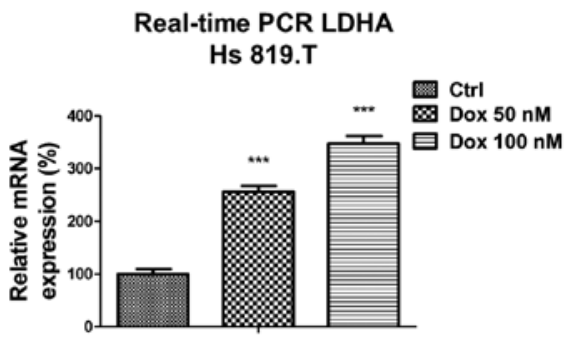

C

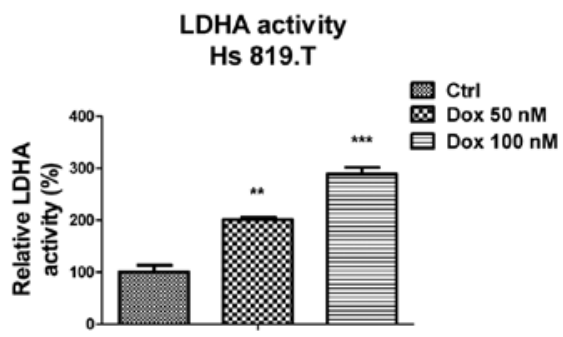

SW1353

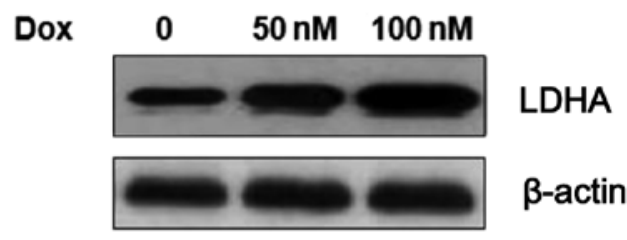

Real-time PCR LDHA

SW1353
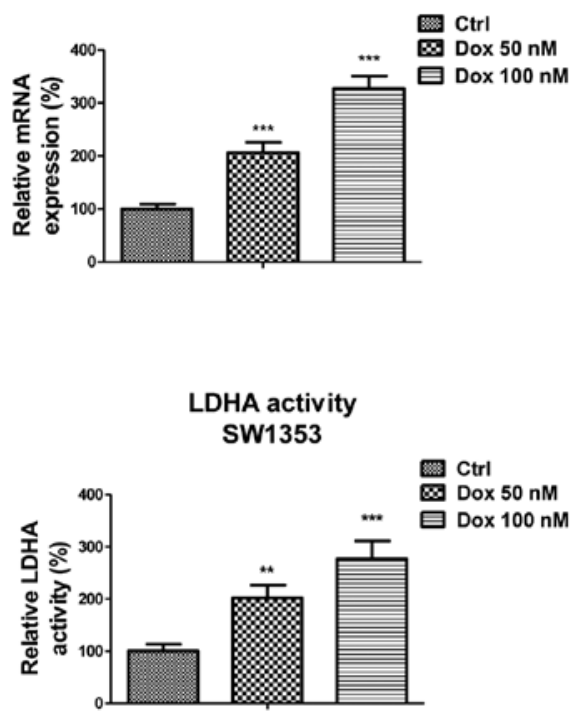

Figure 2. Doxorubicin induces LDHA expression and activity. (A) Chondrosarcoma cells Hs 819.T and SW1353 were treated by doxorubicin at 50 and $100 \mathrm{nM}$ for $72 \mathrm{~h}$ followed by western blot analysis. (B) Chondrosarcoma cells Hs 819.T and SW1353 were treated by doxorubicin at 50 and $100 \mathrm{nM}$ for $72 \mathrm{~h}$ followed by real-time PCR analysis. (C) The activities of LDHA were induced by the indicated doxorubicin treatments for $72 \mathrm{~h}$ under regular cell culture conditions. Columns, mean of three independent experiments; bars, SE. ${ }^{* *} \mathrm{P}<0.01 .{ }^{* * *} \mathrm{P}<0.001$.

through Oncomine.com revealed the expression of LDHA was upregulated in previously published chondrosarcoma microarray database (Fig. 1D). We further compared the activities of LDHA in chondrosarcoma cells and normal chondrocytes. As we expected, the activities of LDHA were higher in malignant chondrosarcoma cells than in normal chondrocytes (Fig. 1E). Taken together, our results suggested the increased activity of LDHA might be an important biomarker for the clinical treatment of chondrosarcoma patients.

Doxorubicin treatment at low-toxic dosage induces LDHA expression and activity. It has been reported that doxorubicin acts as a chemotherapeutic agent against chondrosarcoma cells (17). However, resistance to doxorubicin represents a major obstacle to successful treatment. To explore the biological significance of elevated LDHA in chondrosarcoma cells, we treated chondrosarcoma cells with doxorubicin at a low concentration which does not induce apoptosis sharply for $72 \mathrm{~h}$ followed by the measurement of LDHA expression. Fig. 2A shows the protein expressions of LDHA were significantly upregulated by doxorubicin treatments at low-toxic concentrations in two chondrosarcoma cell lines. The mRNA levels as well as the kinase activities of LDHA in response to multiple doxorubicin treatments were also upregulated (Fig. 2B and C) suggesting LDHA and glucose metabolism are involved in chemotherapy in chondrosarcoma cells.

Doxorubicin-resistant chondrosarcoma cells are more dependent on glucose. To further explore the biological significance of elevated LDHA in chondrosarcoma cells, we generated doxorubicin-resistant cell line using SW1353 parental cells by gradually increasing concentrations of doxorubicin in cell culture medium for a selection of resistant cells. After successive treatments for a duration of three months, several doxorubicin-resistant cell clones were developed and pooled for the following experiments. To verify the resistance, parental cells and resistant pool cells were treated with doxorubicin at multiple concentrations for $72 \mathrm{~h}$. As expected, cell viability assays showed that SW1353 doxorubicin-resistant cells could tolerate much higher concentrations of doxorubicin compared with sensitive cells which exhibited significant inhibition of viability at 100 and $200 \mu \mathrm{M}$ (Fig. 3A). As our above results showed glucose metabolism was highly correlated with doxorubicin treatments (Fig. 3), we investigated the susceptibility of doxorubicin-resistant cells under glucose starvation. SW1353 doxorubicin-resistant cells exhibited more sensitivity to glucose depletion compared with SW1353 parental cells (Fig. 3B). Under low glucose conditions, cell viability of 
A

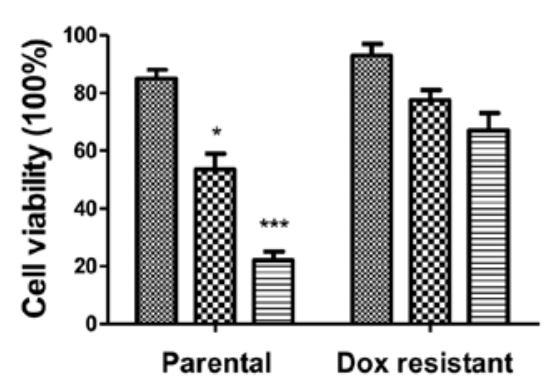

B

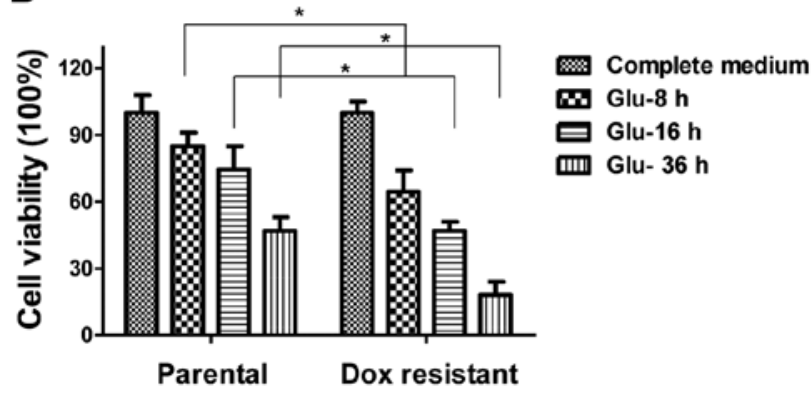

Figure 3. Doxorubicin-resistant chondrosarcoma cells are sensitive to glucose starvation. (A) Generation of doxorubicin-resistant cells. SW1353 cells were treated with gradually increasing concentrations of doxorubicin in regular cell culture conditions for the selection of resistant cells. SW1353 doxorubicinresistant clones were pooled and analyzed by the treatments of doxorubicin at indicated concentrations for $72 \mathrm{~h}$ and were then analyzed by the cell viability assay. (B) Doxorubicin-resistant cells and parental cells were cultured under glucose depletion medium for 8,16 and $36 \mathrm{~h}$ and were then analyzed by cell viability assay. Columns, mean of three independent experiments; bars, $\mathrm{SE}$. ${ }^{*} \mathrm{P}<0.05 .{ }^{* * *} \mathrm{P}<0.001$.

A

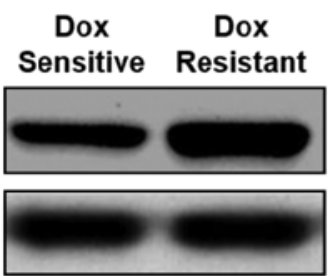

LDHA

$\beta$-actin
Dox $10 \mu \mathrm{M}$ $\mathbb{D} \operatorname{Dox} 100 \mu \mathrm{M}$ Dox $200 \mu \mathrm{M}$
B

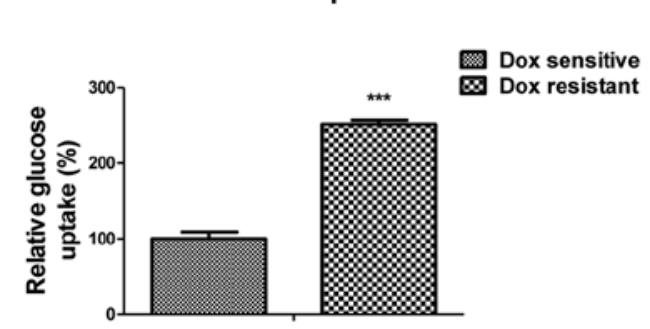

Real-time PCR LDHA

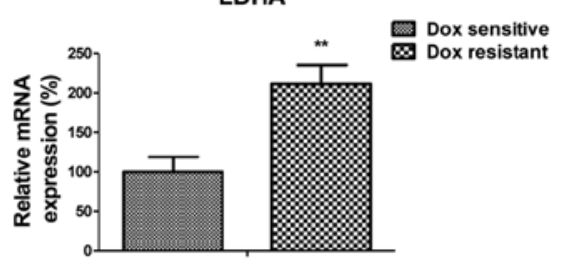

LDHA activity

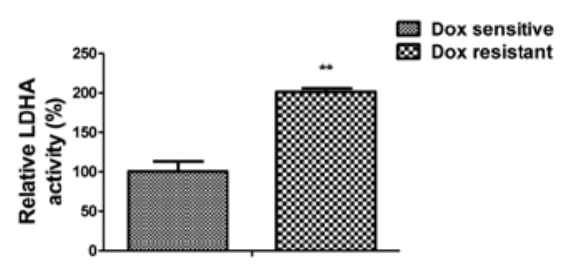

Figure 4. Doxorubicin-resistant cells exhibit upregulated LDHA and glucose metabolism. (A) Western blotting (left panel), real-time PCR (middle panel) and LDHA activity results showed the expression and activity of LDHA were upregulated in doxorubicin-resistant cells. $\beta$-actin was a loading control. (B) Glucose uptake and lactate product were increased in doxorubicin-resistant cells. Columns, mean of three independent experiments; bars, $\mathrm{SE}$. ${ }^{* *} \mathrm{P}<0.01{ }^{* * *} \mathrm{P}<0.001$.

doxorubicin-resistant cells was decreased more than parental cells $(\sim 25 \%)$, suggesting the glucose metabolism in resistant cells was higher than in sensitive cells and might be targets for clinical therapeutic agents.

Increased expression and activity of LDHA in doxorubicinresistant cells. To examine the role of LDHA in mediating doxorubicin resistance in human chondrosarcoma cells, the expression of LDHA was examined in SW1353 parental and doxorubicin-resistant cells (DoxR). We found that LDHA levels were markedly increased in SW1353 DoxR cells, compared to their parental cells (Fig. 4A, left panel). The mRNA and activity of LDHA were also increased $\sim 2$-fold in resistant cells, compared to parental cells (Fig. 4A, middle and right panel). These results indicated that doxorubicin resistance was correlated with the increased LDHA expression and activity.
Notably, SW1353 DoxR cells showed upregulated glucose metabolism. The glucoses uptake and lactate product were significantly increased in resistant cells (Fig. 4B) indicating the metabolic changes contributed to doxorubicin resistance in chondrosarcoma cells.

Inhibition of LDHA re-sensitizes doxorubicin-resistant cells. The increased LDHA expression and LDHA activity detected in doxorubicin-resistant cells suggested that LDHA might play a critical role in doxorubicin resistance. We hypothesized that the downregulation of LDH-A by siRNA might re-sensitize resistant cells to doxorubicin. Therefore, to further verify the effects of LDHA downregulation in the doxorubicin-induced chemotherapy, we knocked down LDHA by specific siRNA in SW1353 parental cells and DoxR cells (Fig. 5A). Consistently, knockdown of LDHA significantly enhanced the sensitivity 
A SW1353 Parental

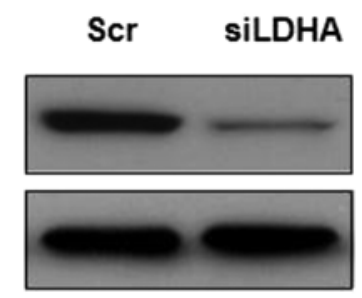

B

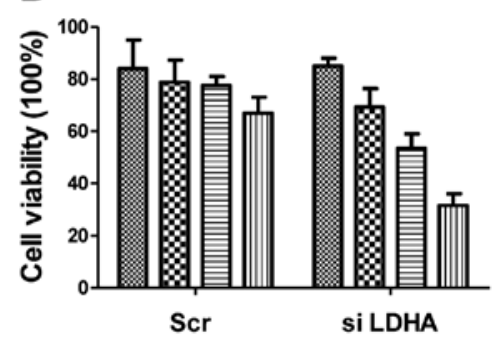

SW1353 Dox R

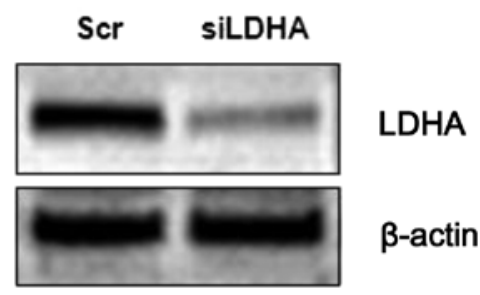

Figure 5. Knockdown of LDHA by siRNA re-sensitizes chondrosarcoma cells to doxorubicin. (A) Knockdown of LDHA by siRNA in SW1353 parental and doxorubicin-resistant cells. $\beta$-actin was a loading control. (B) Knockdown of LDHA in both SW1353 parental (left panel) and doxorubicin-resistant (right panel) cells increased the sensitivity to doxorubicin. Columns, mean of three independent experiments; bars, SE.

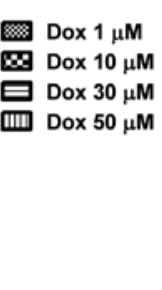

SW1353 Dox R

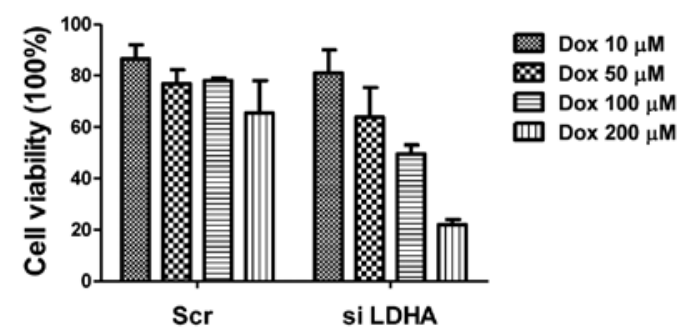

A

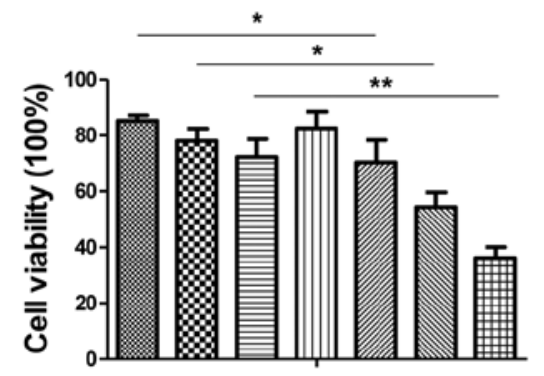

B SW1353
Parental

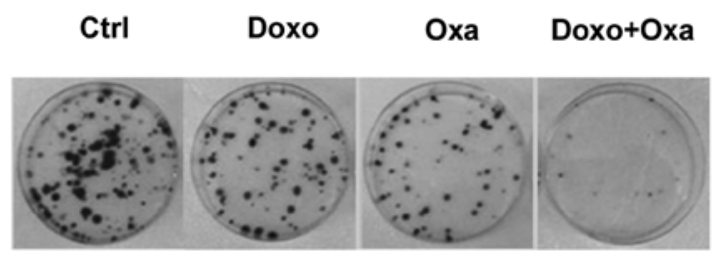

SW1353 Parental

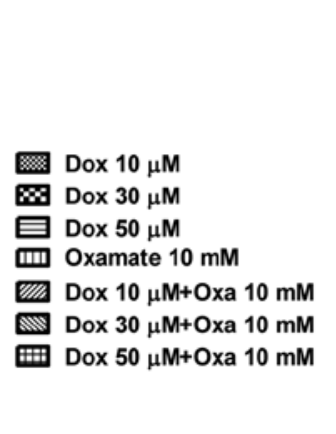

Figure 6. The combination of doxorubicin and oxamate shows synergistic inhibitory effects on chondrosarcoma cells. (A) SW1353 parental (left) and doxorubicin-resistant (right) cells were treated with doxorubicin alone, oxamate alone and combination of doxorubicin with oxamate at the indicated concentration for $72 \mathrm{~h}$ followed by the cell viability analysis. (B) Colony formation assay analysis of SW1353 parental (left panel) and resistant (right panel) cells. Five hundred cells of each experiment were seeded on a $10-\mathrm{cm}$ dish with indicated drugs in regular cell culture medium. The medium and drugs were refreshed every two days. Cells were grown for 3 weeks and the surviving colonies were stained with gentian violet after methanol fixation, and visible colonies ( $>50$ cells) were counted. Colonies from randomly-selected image areas of three replicate wells were enumerated. Columns, mean of three independent experiments; bars, SE. ${ }^{*} \mathrm{P}<0.05 .{ }^{* *} \mathrm{P}<0.01$.

of chondrosarcoma cells to doxorubicin treatments in both parental and resistant cells (Fig. 5B). Knockdown of LDHA decreased the cell viability in parental cells by $30 \%$ at $30 \mu \mathrm{M}$

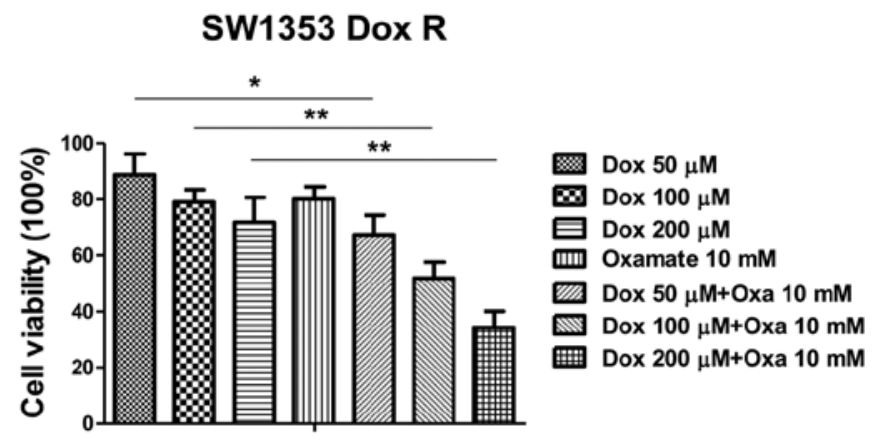

SW1353

Dox R

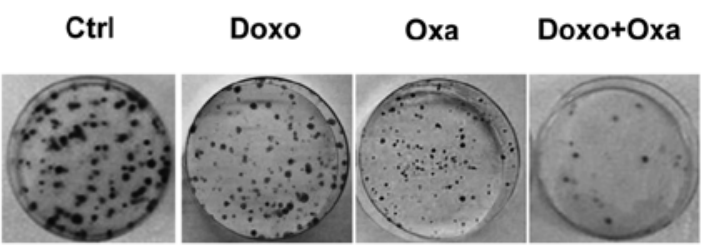


A

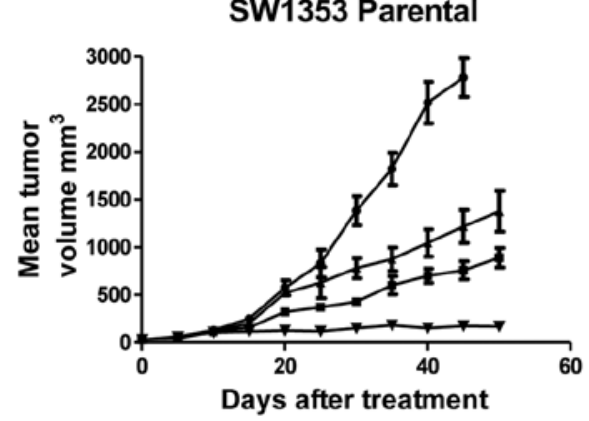

B

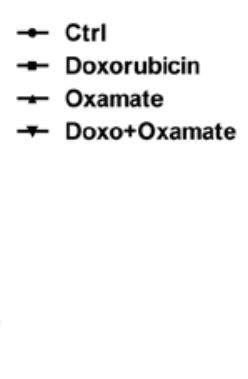

SW1353 Dox Resistant

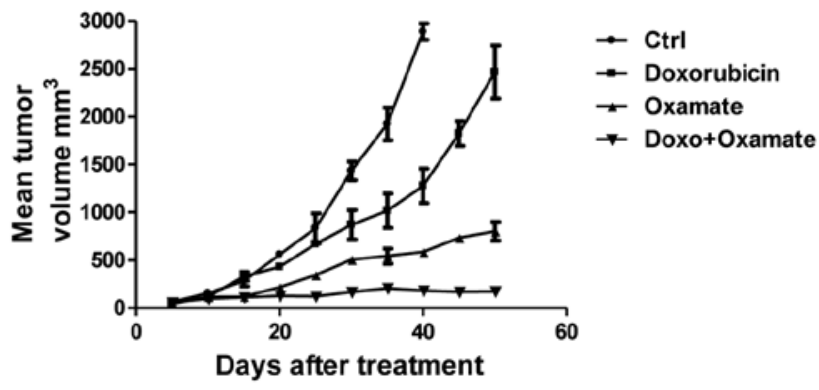

Figure 7. In vivo experiments demonstrate the combination of doxorubicin and glycolysis inhibitors has synergistic effects on the inhibition of chondrosarcoma growth. (A) Mice were inoculated SW1353 parental cells and (B) SW1353 doxorubicin-resistant cells. Once the tumor established, mice were treated with control, doxorubicin alone, oxamate alone and doxorubicin plus oxamate for eight consecutive weeks.

an important role in doxorubicin resistance of chondrosarcoma cells, serving as a promising therapeutic target for overcoming doxorubicin resistance.

The combination of glycolysis inhibitor with doxorubicin shows synergistic inhibitory effects on chondrosarcoma cells in vitro and in vivo. Oxamate is a pyruvate analog that directly inhibits the converting process of pyruvate to lactate by LDHA to inhibit cell glycolysis (16). Since downregulation of LDHA by siRNA significantly inhibited the viability of the doxorubicin-resistant chondrosarcoma cells, we further investigated the effects of combining doxorubicin with glycolysis inhibitor oxamate on the treatment of doxorubicinresistant chondrosarcoma cells. In both SW1353 parental cells and doxorubicin-resistant cells, doxorubicin combined with oxamate was considerably more effective in inhibiting cell viability compared with either agent administered alone (Fig. 6A). To further strengthen our results from the cell viability assay, we performed colony formation assay for the detection of clonogenicity of chondrosarcoma cells under treatments of doxorubicin alone, oxamate alone and the combination of doxorubicin and oxamate. Consistent results from Fig. 6B show significantly increased colony formation inhibition in fourteen days under the treatments of the combination of oxamate and doxorubicin compared with control and treatment with doxorubicin or oxamate alone.

To verify our in vitro results that inhibition of LDHA by siRNA and inhibitor resulted in the re-sensitization of doxorubicin-resistant cells to doxorubicin, we studied the effects of the treatments by the combination of doxorubicin with oxamate on the xenograft tumor growth in nude mice. We injected SW1353 parental cells or DoxR cells into nude mice and treated mice with doxorubicin alone, oxamate alone and doxorubicin with oxamate. After five weeks, mice with the combination treatment had significantly attenuated tumor sizes compared with the treatment alone (Fig. 7), indicating the combination of doxorubicin and oxamate result in strong tumor growth inhibition. Collectively, these results obtained from both in vitro and in vivo experiments established that the combination of doxorubicin with LDHA inhibitor has a greater capacity to inhibit doxorubicin-resistant chondrosarcoma cells compared to either agent alone and might lead to a therapeutic aspect for human chondrosarcoma patients.

\section{Discussion}

Approximately $20 \%$ of skeletal system cancers are chondrosarcomas derived from transformed cells that produce cartilage (1). However, conventional chemotherapy has very limited efficacy in patients with advanced chondrosarcoma and it is not considered active treatment in clinical trial in addition to surgery which is the primary treatment for this chondrosarcoma $(2,3)$. To date, the highest benefit from chemotherapy observed is in mesenchymal and dedifferentiated chondrosarcoma. Therefore, developments of effective and low-toxic therapeutic approaches are required to improve chondrosarcoma clinical management. Evasion of programmed cell death or apoptosis has been recognized as one of the main alterations that dictate malignant growth and is a hallmark of most types of cancer. In the present study, we first reported glucose metabolism is highly active in chondrosarcoma cells compared with normal chondrocytes which may lead us to induce cancer cell apoptosis by inhibiting the key enzymes in the glucose metabolism pathway.

Doxorubicin is frequently used in chemotherapy for a variety of solid tumors, but the efficacy of doxorubicin treatment is limited by drug resistance. Multiple mechanisms responsible for the drug-resistant phenotype in cancer cells have been recognized. The most common is characterized by the enhanced expression of the P-glycoprotein, ABCB1 which is a transmembrane pump responsible for drug efflux from cells (18). Recently, another study described that loss of HuR, which is an RNA binding protein involved in the post-transcriptional regulation of a wide spectrum of mRNAs, is responsible for the doxorubicin resistance in breast cancer (19). Restoration of HuR expression in breast cancer cells re-sensitized resistant cells to doxorubicin (19). Therefore, the purpose of the present study was to find new, targeted treatment strategies to overcome chemoresistance for clinical chondrosarcoma patients.

Cancer cells depend mostly on glycolysis, the anaerobic breakdown of glucose into the energy-storing molecule ATP, even in the presence of available oxygen while normal cells rely primarily on the process of mitochondrial oxidative phosphorylation (11). Therefore, we sought to use these unique bioenergetic properties to improve the therapeutic efficacy to inhibit cancer cells. The combination of Taxol and oxamate was previously reported to enhance the therapeutic effects on 
human breast cancer cells (16). In this study, we investigated the role of LDHA in the acquired doxorubicin resistance in human chondrosarcoma cells. We identified that compared to parental cells, doxorubicin-resistant cells possess an increased expression and activity of LDHA. Downregulation of LDHA resulted in an increased sensitivity of doxorubicin-resistant cells. In addition, compared to parental cells, doxorubicinresistant cells showed a higher sensitivity to the LDHA inhibitor oxamate. Notably, we found inhibition of LDHA significantly inhibited chondrosarcoma cell viability when combined with doxorubicin in vitro and in vivo, demonstrating the important roles of LDHA in overcoming chemoresistance in chondrosarcoma cells. In our next study, we will focus on the mechanisms by which highly active glucose metabolism makes chondrosarcoma cells evade apoptosis in response to doxorubicin treatment. Proteomic approaches have been applied to identify the putative LDHA interaction proteins under the treatment of doxorubicin, and, more importantly, we will explore more novel glycolysis inhibitors which contribute to the therapeutic effects on the treatment of chondrosarcoma patients. In general, the results of the present study demonstrated that LDHA plays an important role in doxorubicin resistance and that it may potentially serve as a therapeutic target for overcoming chemoresistance in chondrosarcoma patients.

\section{Acknowledgements}

The authors thank the staff and faculty working in the Department of Orthopaedics, The 101st Hospital of the People's Liberation Army. We thank Dr Xiangyong Li from the Department of Hematology and Oncology, The 101st Hospital of the People's Liberation Army for the editorial assistance.

\section{References}

1. Gelderblom H, Hogendoorn PC, Dijkstra SD, van Rijswijk CS, Krol AD, Taminiau AH and Bovée JV: The clinical approach towards chondrosarcoma. Oncologist 13: 320-329, 2008.

2. Fiorenza F, Abudu A, Grimer RJ, Carter SR, Tillman RM, Ayoub K, Mangham DC and Davies AM: Risk factors for survival and local control in chondrosarcoma of bone. J Bone Joint Surg Br 84: 93-99, 2002.

3. Italiano A, Mir O, Cioffi A, Palmerini E, Piperno-Neumann S, Perrin C, Chaigneau L, Penel N, Duffaud F, Kurtz JE, Collard O, Bertucci F, Bompas E, Le Cesne A, Maki RG, Ray Coquard I and Blay JY: Advanced chondrosarcomas: role of chemotherapy and survival. Ann Oncol 24: 2916-2922, 2013.

4. Onishi AC, Hincker AM and Lee FY: Surmounting chemotherapy and radioresistance in chondrosarcoma: molecular mechanisms and therapeutic targets. Sarcoma 2011: 381564, 2011.

5. Perez J, Decouvelaere AV, Pointecouteau T, Pissaloux D, Michot JP, Besse A, Blay JY and Dutour A: Inhibition of chondrosarcoma growth by mTOR inhibitor in an in vivo syngeneic rat model. PLoS One 7: e32458, 2012.
6. Wang S, Konorev EA, Kotamraju S, Joseph J, Kalivendi S and Kalyanaraman B: Doxorubicin induces apoptosis in normal and tumor cells via distinctly different mechanisms: intermediacy of $\mathrm{H}_{2} \mathrm{O}_{2}$ - and p53-dependent pathways. J Biol Chem 279: 25535-25543, 2004.

7. Finn NA, Findley HW and Kemp ML: A switching mechanism in doxorubicin bioactivation can be exploited to control doxorubicin toxicity. PLoS Comput Biol 7: e1002151, 2011.

8. Gilliam LA, Moylan JS, Patterson EW, Smith JD, Wilson AS, Rabbani Z and Reid MB: Doxorubicin acts via mitochondrial ROS to stimulate catabolism in $\mathrm{C} 2 \mathrm{C} 12$ myotubes. Am J Physiol Cell Physiol 302: C195-C202, 2012.

9. Efferth T, Giaisi M, Merling A, Krammer PH and Li-Weber M: Artesunate induces ROS-mediated apoptosis in doxorubicinresistant T leukemia cells. PLoS One 2: e693, 2007.

10. Zhang J, Zhou F, Wu X, Zhang X, Chen Y, Zha BS, Niu F, Lu M, Hao G, Sun Y, Sun J, Peng Y and Wang G: Cellular pharmacokinetic mechanisms of adriamycin resistance and its modulation by 20(S)-ginsenoside Rh2 in MCF-7/Adr cells. Br J Pharmacol 165: 120-134, 2012.

11. Vander Heiden MG, Cantley LC and Thompson CB: Understanding the Warburg effect: the metabolic requirements of cell proliferation. Science 324: 1029-1033, 2009.

12. Liu Y, Cao Y, Zhang W, Bergmeier S, Qian Y, Akbar H, Colvin R, Ding J, Tong L, Wu S, Hines J and Chen X: A small-molecule inhibitor of glucose transporter 1 downregulates glycolysis, induces cell-cycle arrest, and inhibits cancer cell growth in vitro and in vivo. Mol Cancer Ther 11: 1672-1682, 2012.

13. Cao X, Fang L, Gibbs S, Huang Y, Dai Z, Wen P, Zheng X, Sadee W and Sun D: Glucose uptake inhibitor sensitizes cancer cells to daunorubicin and overcomes drug resistance in hypoxia. Cancer Chemotherapy Pharmacol 59: 495-505, 2007.

14. Nakano A, Tsuji D, Miki H, Cui Q, El Sayed SM, Ikegame A, Oda A, Amou H, Nakamura S, Harada T, Fujii S, Kagawa K, Takeuchi K, Sakai A, Ozaki S, Okano K, Nakamura T, Itoh K, Matsumoto T and Abe M: Glycolysis inhibition inactivates ABC transporters to restore drug sensitivity in malignant cells. PloS One 6: e27222, 2011.

15. Zhou GQ, Tang LL, Mao YP, Chen L, Li WF, Sun Y, Liu LZ, Li L, Lin AH and Ma J: Baseline serum lactate dehydrogenase levels for patients treated with intensity-modulated radiotherapy for nasopharyngeal carcinoma: a predictor of poor prognosis and subsequent liver metastasis. Int J Radiat Oncol Biol Phys 82: e359-e365, 2012.

16. Zhou M, Zhao Y, Ding Y, Liu H, Liu Z, Fodstad O, Riker AI, Kamarajugadda S, Lu J, Owen LB, Ledoux SP and Tan M: Warburg effect in chemosensitivity: targeting lactate dehydrogenase-A re-sensitizes taxol-resistant cancer cells to taxol. Mol Cancer 9: 33, 2010.

17. Van Oosterwijk JG, Herpers B, Meijer D, Briaire-de Bruijn IH, Cleton-Jansen AM, Gelderblom H, van de Water B and Bovée JV: Restoration of chemosensitivity for doxorubicin and cisplatin in chondrosarcoma in vitro: BCL-2 family members cause chemoresistance. Ann Oncol 23: 1617-1626, 2012.

18. Zaja R, Caminada D, Loncar J, Fent K and Smital T: Development and characterization of P-glycoprotein 1 (Pgp1, ABCB1)mediated doxorubicin-resistant PLHC-1 hepatoma fish cell line. Toxicol Appl Pharmacol 227: 207-218, 2008.

19. Latorre E, Tebaldi T, Viero G, Spartà AM, Quattrone A and Provenzani A: Downregulation of HuR as a new mechanism of doxorubicin resistance in breast cancer cells. Mol Cancer 11: 13, 2012. 